\title{
Design of Miniaturized Printed Antenna for Mobile Phones
}

\author{
Tobin Thomas ${ }^{1}$, Jinu Baby ${ }^{2}$ \\ ${ }^{I} M$ Tech Student, ${ }^{2}$ Assistant Professor,,${ }^{1,2}$ Department of Electronics and Communication Engineering, Mar \\ Baselios College of Engineering and Technology, Kerala, India
}

\begin{abstract}
With the rapid growth of communication technologies, mobile communications require a mobile phone to be operated in various communication services. This has led to a great demand for designing antennas with some desirable features such as multiband operation, low specific absorption rate (SAR), light weight, and low profile. The mutual interactions between the mobile terminals and the human body are to be considered. A part of the electromagnetic wave radiated by the antenna is absorbed by the human head. Some mobile hand set antenna characteristics, such as radiation pattern, radiation efficiency, bandwidth, and return loss are altered due to the proximity of the human head. Different methods to reduce the SAR produced by a handset antenna were employed. Disadvantage of these method is the increasing the size and cost of the antenna. Effort is to minimize mobile handset antenna size and cost with increasing the services provided by the antenna. An internal antenna consisting of a monopole with a meander line and an EBG structure is embedded on the bottom layer of the substrate to reduce SAR and to cover multibands including the LTE bands. This work presents a technique to reduce SAR of mobile handset antenna by using the meander line. The simulation is carried out in CST tool using both EBG method and split ring resonator method. The simulation results indicate that the proposed scheme using split ring resonator method outperforms the EBG method in order to reduce the SAR of mobile hand set.
\end{abstract}

Index Terms: Antenna, SAR, EBG, Radiation pattern, gain, SRR

\section{Introduction}

In mobile phone systems or any other wireless systems, antennas are the key technology. In a wireless system, an antenna converts guided radio wave energy (such as a signal traveling in a coaxial cable for television) to energy that is emitted or "radiated" out into free space. An antenna also does the reverse-it receives radio waves from the air and feeds them into the devices that detect, decode, and amplify them. In a cell phone system, there is one antenna in the handset and another in the base station tower. Both of these antennas transmit and receive waves. Base station antennas are the long, narrow rectangular devices, usually in groups of three, mounted high on a base station tower. The base station can be thought of as the "command center" that both sends and receives signals to the consumer's hand set [1]. The base station performs a "router" function to properly direct incoming and outgoing calls, and a "repeater" function to enable the consumer to communicate from cell to cell within the terrestrial cellular network. The base station antenna is mounted on tall towers because from this high point it is easier to stay in communication with cell phone users, who are often near the ground.

When designing cellular phone antennas, especially the ones for the base stations, engineers have to balance several different needs and concerns [2]. The first is "directionality." All transmitting antennas radiate energy in a particular pattern that depends on the shape of the antenna and other factors. It is best for a cellular system if that pattern is roughly parallel to the ground, where most cell phone users are located. Another important concern is called "gain," which refers to the fact that an antenna does not transmit (or receive) waves from every direction with equal sensitivity. A third factor is physical size. Because antennas have to be placed along roads and in other public places, they cannot be too tall or too large. Engineers have to find compromises that take into account these and other factors to design antennas that work well.

A cellular handset antenna is the small cylindrical stub sticking out of the top of the hand set case. In some cases antennas are embedded in the handset case and are not visible to the user. Cell phone handset antennas pose a different set of problems than the base stations. Since a cell phone user is constantly changing his or her position and moving from cell to cell, the mobile phone requires an antenna that transmits and receives equally well in all directions. Unfortunately, an antenna of this type is always less sensitive than one optimized to transmit or receive in a narrow beam. Engineers must design the antenna so that it provides both good mobility and good voice quality.

Another major concern in cellular antenna design is the potential radiation hazards posed to humans. As the use of cell phones increases, there are growing concerns about what affect the cell phone's radio waves have on human health[3]. Various antenna designs have been promoted to minimize the amount of energy that is radiated into the skull of the user. 
The specific absorption rate (SAR) is a defined figure of merit to evaluate the power absorbed by biological tissues[4].The SAR values are calculated according to the 10-g standard of the human tissue mass. The SAR calculations are done using the CST 2012 commercial package with Hugo model CST Microwave Studio; the tissues that are contained have relative permittivities and conductivities. The tissues and frequency dispersive properties are taken into consideration [5]. The averaged 10-g SAR is at the afore-mentioned operating frequencies when the antenna is in close proximity to the body.

The use of the EBG structure miniaturizes the size, widens the bands, and reduces the SAR values.

\section{EBG Antenna design}

The EBG structure allows to manipulate the propagation of electromagnetic waves also it radiates the signal into an elevated plane. The EBG structures interacts with the electromagnetic waves, shows amazing properties such as frequency pass bands, stop bands, or band-gaps. The characteristics of the EBG structure are tested by a microstrip line over one column of the EBG unit with a 0.5-mm gap. The proposed EBG configuration reveals stop bands at most of the mobile applications bands. This means that it has high surface impedance within these bands, where the tangential magnetic field is small, even with a large electric field along the surface. The EBG structure is positioned perpendicular to the two antennas, the monopole, and the meander line. With the existence of the EBG, the EBG structure acts as an artificial magnetic conductor, AMC, within its stop bands. The AMC enhances the radiation in the direction opposite to the position of the human body. Thus, it lowers the SAR absorbed within the human tissues. It is evident that the EBG enhances the radiation in the elevation plan.

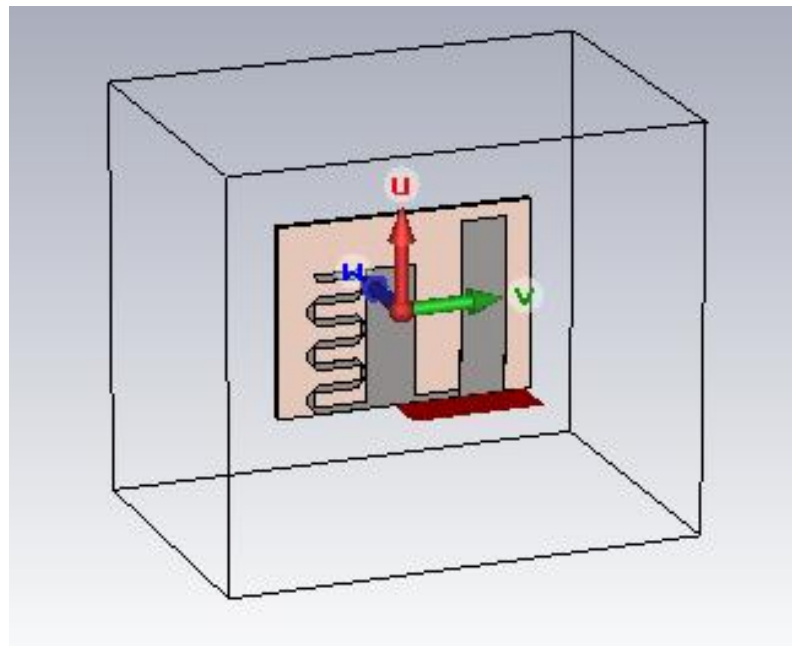

Fig. 1. Front view of EBG Antenna design.

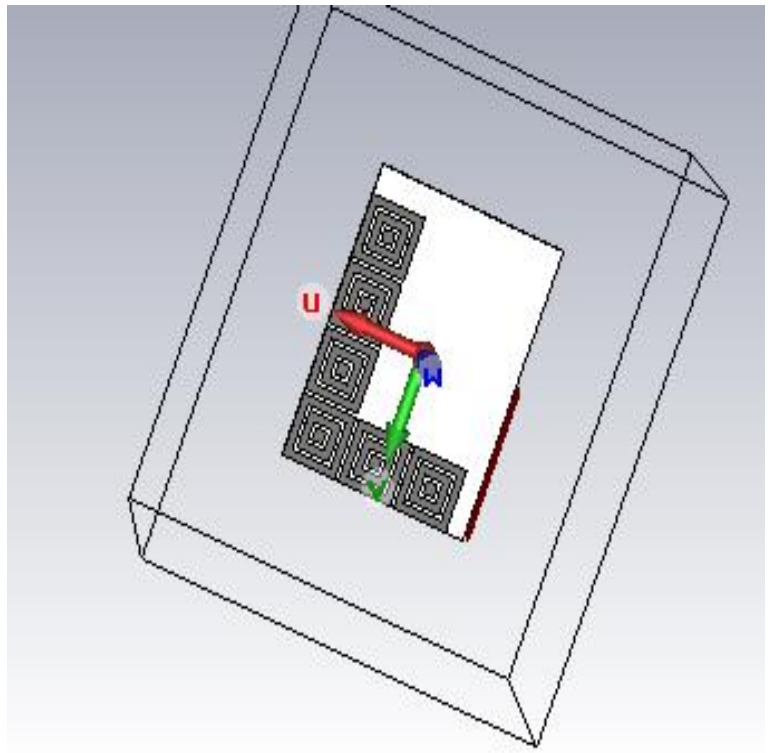

Fig. 2.Back view of EBG Antenna design 


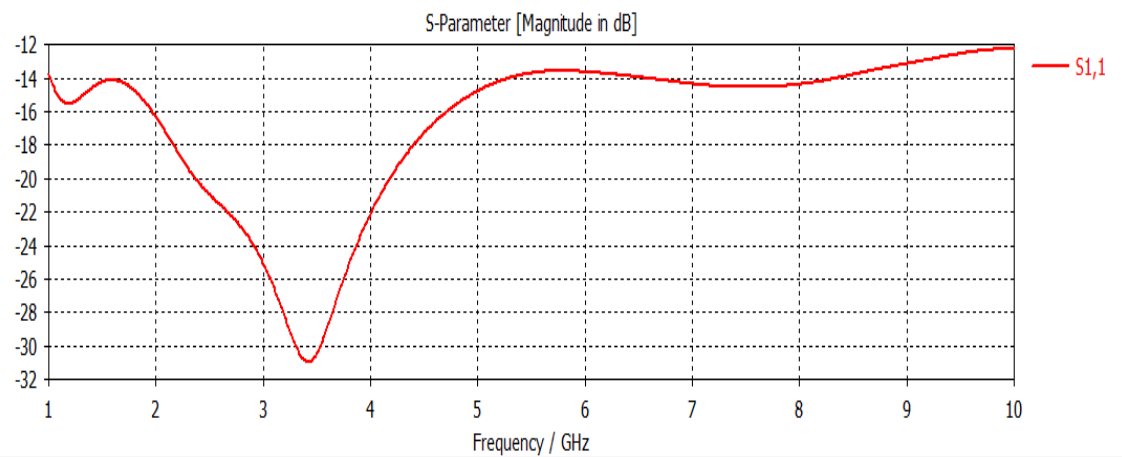

Fig. 3 S - Parameter graph of SRR Antenna design

Farfield Gain Abs (Phi=90)

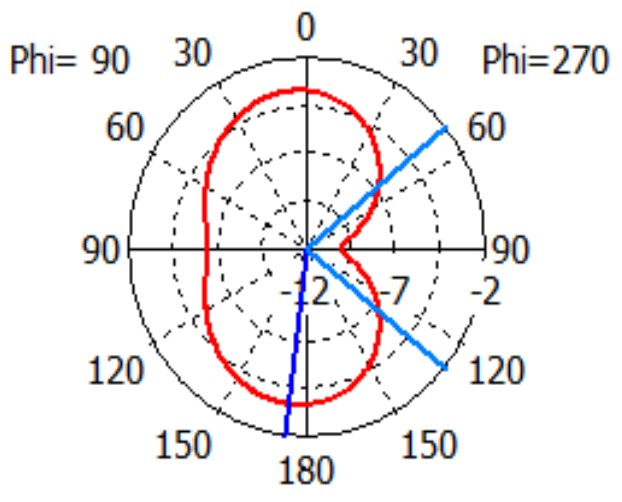

Theta / Degree vs. dB

Fig. 4. Radiation pattern in XY plane

Farfield Gain Abs (Phi=90)

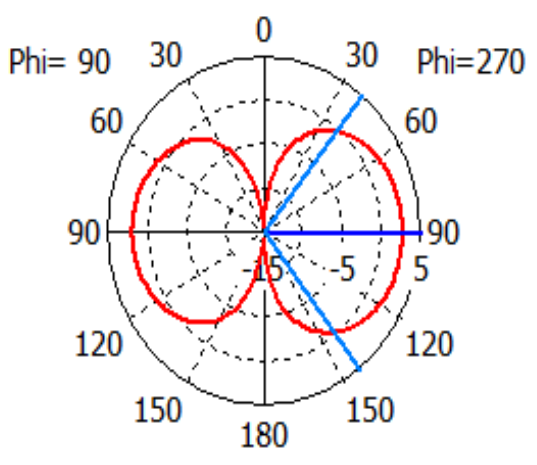

Theta / Degree vs. dB

Fig. 5. Radiation pattern in XY plane

\section{Proposed SRR Antenna design}

Split ring resonators (SRRs) design is used to produce the negative dielectric constant or permittivity and negative permeability. The split ring resonator can be designed in different shapes. There are many types of split ring resonator that have been designed and proposed by researchers. Edge coupled SRR (EC-SRR) was the initial first design by Pendry. In this SRR design, there is a metallic split ring printed on the conducting path. The complementary split ring resonator structure (CSRR) is obtained by replacing the copper area with substrate material. 


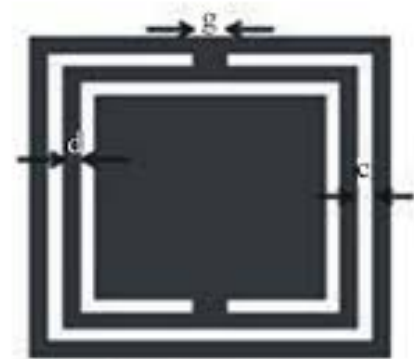

Metallization

Substrate

Fig. 6. Unit cell of SRR

Farfield E-Field $(r=1 \mathrm{~m})$ Abs (Theta $=90$ )

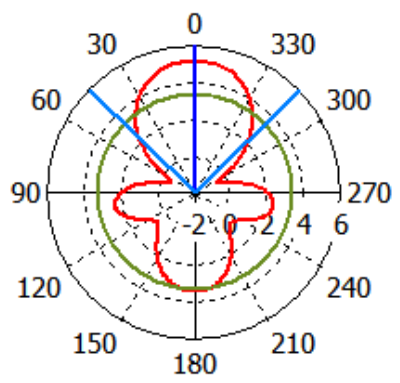

Phi / Degree vs. dBV/m

Fig. 7. Radiation Pattern

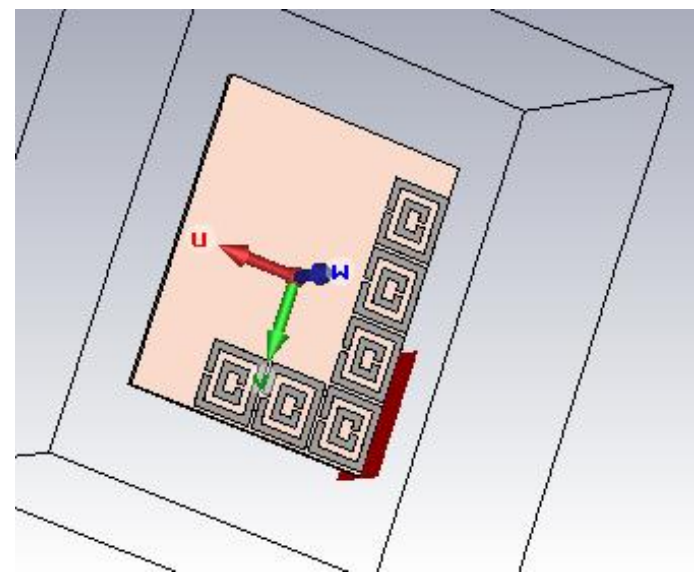

Fig. 8. Back view of SRR Antenna design.

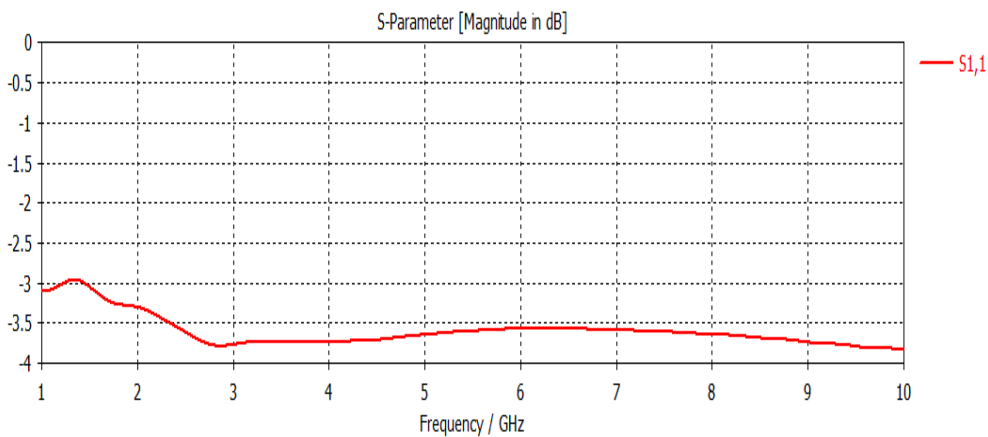

Fig.9 S - Parameter graph of SRR Antenna design 


\begin{tabular}{|l|l|l|}
\hline \multirow{2}{*}{ Frequency $(\mathrm{GHz})$} & \multicolumn{2}{|l|}{ SAR Value $(\mathrm{W} / \mathrm{Kg})$} \\
\cline { 2 - 3 } & EBG & SRR \\
\hline 0.7 & 1.77512 & 1.5243 \\
\hline 0.9 & 1.412 & 1.322 \\
\hline 1.5 & 0.7681 & 0.7516 \\
\hline 2 & 0.851 & 0.817 \\
\hline
\end{tabular}

Table. 1. Comparison of SAR values between EBG and SRR.

\section{Conclusion}

A new compact planar antenna design that supports all of the operating mobile services, ISM applications, and wireless communication services has designed. The use of the SRR structure miniaturizes the size, widens the bands, and reduces the SAR values. The SAR values of the antenna satisfy the standard safety guidelines. The SAR values and the power absorption are not strictly related, because an antenna may have a high SAR value while its efficiency remains similar, without suffering from the head's absorption. The SAR measurements give information about the electric-field peaks being absorbed by the head. The ratio between the antenna's efficiency and the SAR value at each frequency and for each antenna position is different. The effect of the human body on the antenna performance should also taken into consideration. The antenna should have more compact size and better performance when compared to other published antennas.

\section{References}

[1]. K. S. Sultan, H. H. Abdullah, E. A. Abdallah, and E. A. Hashish, "Low-SAR, Miniaturized Printed Antenna for Mobile, ISM, and WLAN Services",IEEE Antennas And Wireless Propagation Letters, vol. 12, 2013.

[2]. C. Picher, J. Anguera, A. Andujar, C. Puente, and S. Kahng, "Analysis of the human head interaction in handset antennas with slotted ground planes," IEEE Antennas Propag. Mag., vol. 54, no. 2, pp. 36-56, Apr.2012.

[3]. S. I. Kwak, D. Sim, and J. H. Kwon, "Design of optimized multilayer PIFA with the EBG structure for SAR reduction in mobile applications,"IEEE Trans. Electromagn. Compat., vol. 53, no. 2, pp. 325-333, May 2011.

[4]. R. G. Villanueva, H. J. Aguilar, and R. L. Miranda, "State of the art methods for low SAR antenna implementation," Proc. EuCAP, pp. $1-4,2010$.

[5]. Yongyan Du and Anping ZhaoJ, “An Internal Quad-Band Antenna for Oval-Shaped Mobile Phones,"IEEE Antennas and Wireless Propagation letters, vol. 9, 2010 